\title{
Pediatric Recurrent Rosai-Dorfman Disease with germline heterozygous SLC29A3 and somatic MAP2K1 mutations
}

Shruthi Suryaprakash ${ }^{1}$, Amy George $^{1}$, Scott Langenburg ${ }^{1}$, and Sureyya Savasan ${ }^{2}$

${ }^{1}$ Children's Hospital of Michigan

${ }^{2}$ Wayne State University School of Medicine

August 25, 2020

Pediatric Recurrent Rosai-Dorfman Disease with germline heterozygous SLC29A3 and somatic MAP2K1 mutations

Shruthi Suryaprakash, $\mathrm{MD}^{1}$, Amy George, $\mathrm{RN}^{2}$, Scott Langenburg, $\mathrm{MD}^{3}$, and Süreyya Savaşan, $\mathrm{MD}^{2,4}$

${ }^{1}$ Department of Pediatrics, Children's hospital of Michigan, Detroit

${ }^{2}$ Division of Hematology/Oncology, Children's hospital of Michigan, Detroit

${ }^{3}$ Division of Pediatric Surgery, Children's hospital of Michigan, Detroit

${ }^{4}$ Division of Hematology/Oncology and Blood and Marrow Transplant Program, Department of Pediatrics, Children's Hospital of Michigan, Barbara Ann Karmanos Cancer Center, Central Michigan University College of Medicine, Detroit

Correspondence: Süreyya Savaşan, MD

3901 Beaubien Blvd.

Division of Hematology/Oncology

Blood and Marrow Transplant Program

Children's Hospital of Michigan

Detroit, Michigan 48201

E-mail: savas1s@cmich.edu

Phone: 313-745-5516 Fax: 313-745-5237

Text word count: 500

Reference count: 16

Tables and figures: 1

Page number: 7

Short running title: Germline and somatic mutations in recurrent Rosai-Dorfman disease

Key words: recurrent RDD, germline SLC29A3 mutation, somatic MAP2K1 mutation, dysgammaglobulinemia, clonal T-LGL proliferation

Abbreviations Table 


\begin{tabular}{ll}
\hline RDD & Rosai Dorfman Disease \\
\hline LCH & Langerhans cell histiocytosis \\
HHV6 & Human herpes virus 6 \\
TCR & T-cell receptor \\
T-LGL & T-cell large granular lymphocyte \\
ALPS & Autoimmune lymphoproliferative syndrome \\
\hline
\end{tabular}

This case has been submitted for presentation at the Histiocyte Society meeting in 2020 .

To the Editor:

Rosai-Dorfman disease (RDD) is a rare non-Langerhans cell histiocytosis (LCH), disorder listed under the 'R group' of histiocytosis including familial and sporadic forms by the Histiocyte Society. ${ }^{1-3}$ Patients with RDD classically present with cervical lymphadenopathy and some have extranodal disease. SomaticKRAS and MAP2K1 mutations were reported in RDD patients. ${ }^{4}$ We report a patient with recurrent RDD with underlying germline and somatic mutations.

A currently 7-year-old African-American male patient presented with cervical lymphadenopathy at 19 months of age. He had dysgammaglobulinemia with increased $\operatorname{IgG}$, $\operatorname{Ig} \mathrm{A}$, and $\operatorname{IgE}$ levels and elevated C-reactive protein and erythrocyte sedimentation rate. He tested positive for human herpesvirus 6 (HHV6). Lymph node biopsy findings were consistent with RDD and negative for HHV6 staining. Due to the progression, he was treated on oral steroids with improvement. He presented 2 1/2 years later with recurrent lymphadenopathy and also had been diagnosed with autism spectrum disorder. Repeat scans and biopsy confirmed the recurrence of RDD (Figure1 A-C). A sizeable population of CD5-dim lymphocytes and clonal T-cell receptor (TCR) rearrangement pattern suggested T-cell large granular lymphocyte (T-LGL) expansion correlating with B-cell population (Figure1-D). He failed intravenous steroids and has had two debulking surgeries so far. Vitamin B12 levels were very high and methylmalonic acid within normal levels. He has decreased lymphocyte responses to phytohemagglutinin, concanavalin A, tetanus and candida antigens with currently normal immunoglobulin levels and small lymphadenopathy. Tumor tissue 596 gene mutational analysis on the most recent sampling, but not on previous ones revealed a known pathologic somatic MAP2K1 gain of function mutation (c.159T >A p.F53L), along with overexpression ofMAP2K1 NFBK1, and NFBK2 mRNA level; whole-exome sequencing showed likely pathogenic germline heterozygous mutations in SLC29A3 (c.45delC) and ACSF3 (c.1075G $>$ A) genes.

This patient has dysgammaglobulinemia, and increased inflammatory markers, which has correlated with disease burden and shown improvements following debulking surgeries. Increased T-LGL (18-41\%) with clonal TCR rearrangement pattern likely reflect persistent immune dysfunction. ${ }^{5}$ Despite very high vitamin B12 levels, a known feature of autoimmune lymphoproliferative syndrome (ALPS) and known link between ALPS and RDD, he neither has increased double-negative TCR alpha/beta T-cells, another hallmark of ALPS nor any known ALPS-related mutations. ${ }^{6-7}$

Homozygous germline SLC29A3 mutations are described in Faisalabad histiocytosis and familial, but not sporadic RDD. ${ }^{8}$ Cases of Faisalabad histiocytosis may mimic RDD. ${ }^{9}$ Mutations in ACSF3 are associated with combined malonic and methylmalonic aciduria and $40 \%$ of the patients develop symptoms in adulthood. ${ }^{10}$

Reactive nature of RDD and possible association with viruses have been suggested. ${ }^{11,12}$ Discovery of BRAFV600E mutations in LCH and Erdheim-Chester disease supported the neoplastic nature of histiocytic disorders. ${ }^{13}$ Mutations in MAP2K1 have been associated with LCH and RDD and mutually exclusive somatic mutations in KRAS and MAP2K1 with RDD. ${ }^{14,4}$ Though somatic MAP2K1 mutation might have been crucial in RDD pathogenesis in this patient, likely pathogenic and heterozygous SLC29A3 mutation might have been contributory as well. Similarly, dysgammaglobulinemia and inflammation process could be related to SLC29A3 mutation. ${ }^{15,16}$ Therefore, this case raises the possibility of cooperating germline and somatic mutations in the development of RDD. 


\section{References}

1. Destombes P. Adenitis with lipid excess, in children or young adults, seen in the Antilles and in Mali (4 cases). Bull Soc Pathol Exot. 1965;58(6):1169-1175.

2. Rosai J, Dorfman RF. Sinus histiocytosis with massive lymphadenopathy. A newly recog- nized benign clinicopathological entity. Arch Pathol. 1969;87(1):63-70.

3. Emile J-F, Abla O, Fraitag S, et al. Revised classification of histiocytoses and neoplasms of the macrophage-dendritic cell lineages. Blood 2016;127:2672-81.

4. Garces S, Medeiros LJ, Patel KP, et al. Mutually exclusive recurrent KRAS and MAP2K1 mutations in Rosai-Dorfman disease. Modern Pathology . 2017;30(10):1367-1377.

5. Savaşan S, Al-Qanber B, Buck S, Wakeling E, Gadgeel M. Clonal T-cell large granular lymphocyte proliferations in childhood and young adult immune dysregulation conditions. Pediatric Blood 83 Cancer . 2020;67(5).

6. Maric I, Pittaluga S, Dale JK, et al. Histologic features of sinus histiocytosis with massive lymphadenopathy in patients with autoimmune lymphoproliferative syndrome. Am J Surg Pathol. 2005;29(7):903-911.

7. Xie Y, Pittaluga S, Price S, et al. Bone marrow findings in autoimmune lymphoproliferative syndrome with germline FAS mutation. Haematologica. 2017;102(2):364-372.

8. Morgan NV, Morris MR, Cangul H, et al. Mutations in SLC29A3, encoding an equili- brative nucleoside transporter ENT3, cause a familial histiocytosis syndrome (Faisalabad histiocytosis) and familial RosaiDorfman disease. PLoS Genet. 2010;6(2):

9. Rossbach H-C, Dalence C, Wynn T, Tebbi C. Faisalabad histiocytosis mimics Rosai-Dorfman disease: Brothers with lymphadenopathy, intrauterine fractures, short stature, and sensorineural deafness. $P e$ diatric Blood $\& 3$ Cancer . 2006;47(5):629-632.

10. Sloan J, Johnston J, Manoli I et al. Exome sequencing identifies ACSF3 as a cause of combined malonic and methylmalonic aciduria. Nat Genet. 2011;43(9):883-886.

11. Paulli M, Bergamaschi G, Tonon L, et al. Evidence for a polyclonal nature of the cell infiltrate in sinus histiocytosis with massive lymphadenopathy (Rosai-Dorfman disease). Br J Haematol. 1995;91(2):415418.

12. Levine PH, Jahan N, Murari P, Manak M, Jaffe ES. Detection of Human Herpesvirus 6 in Tissues Involved by Sinus Histiocytosis with Massive Lymphadenopathy (Rosai-Dorfman Disease). Journal of Infectious Diseases . 1992;166(2):291-295.

13. Haroche J, Charlotte F, Arnaud L, et al. High prevalence of BRAF V600E mutations in ErdheimChester disease but not in other non-Langerhans cell histiocytoses. Blood. 2012;120(13):2700-2703.

14. Zeng K, Ohshima K, Liu Y et al. BRAFV600E and MAP2K1 mutations in Langerhans cell histiocytosis occur predominantly in children. Hematol Oncol. 201a6;35(4):845-851.

15. Melki I, Lambot K, Jonard L, et al. Mutation in the SLC29A3 gene: a new cause of a monogenic, autoinflammatory condition. Pediatrics. 2013;131(4):e1308-e1313.

16. Chela James, Simon Eaton, Paul Brogan \& Khalid Hussain 2011. Pigmented hypertrichosis and insulin dependent diabetes mellitus (PHID) syndrome is associated with chronic inflammation and involves the NF-kB response pathway of inflammation. Presented at Society for Endocrinology BES 2017, London, UK. Endocrine Abstracts 27 P56

\section{Figure Legend}

Figure1. Clinical, imaging and flow cytometric findings in the case. (A) Large right cervical lymphadenopathy; (B) Magnetic resonance imaging showing multiple enlarged lymph nodes forming a conglomerate in right cervical region; (C) Several lymph nodes removed by surgery; (D) While increased peripheral blood CD5-dim T cells (24.9\%) express predominantly CD8 (61\%), some are CD4/CD8-negative (double negative). 

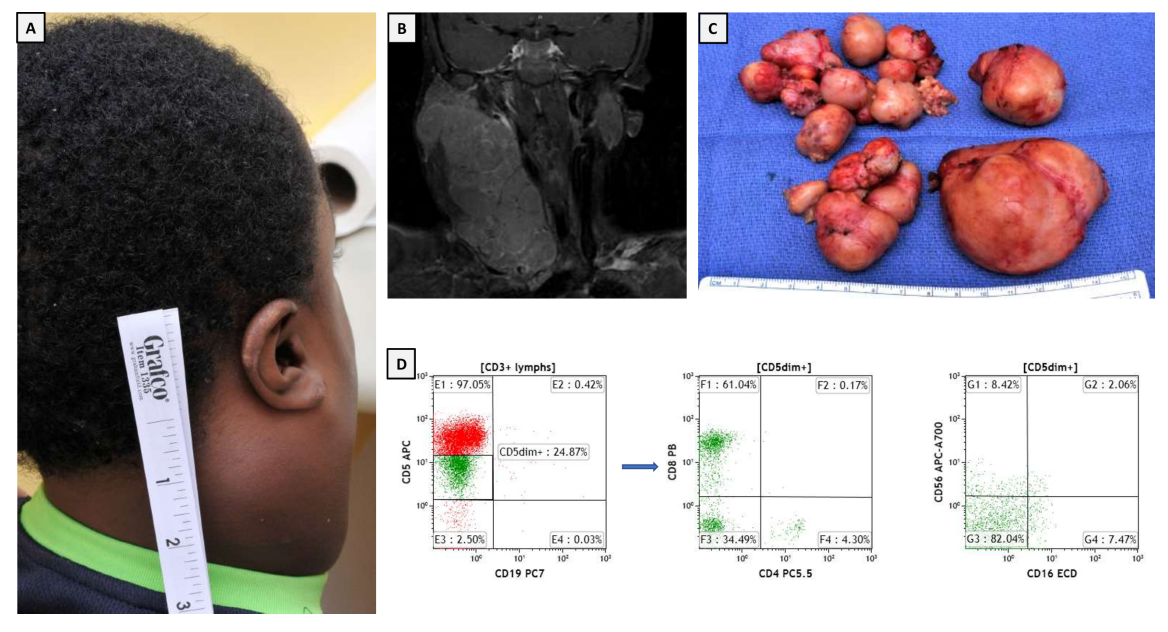